\title{
Debulking Surgery for Pelvic Recurrence After Surgically-treated Tubal Gestational Choriocarcinoma - A Case Report and Literature Review
}

\author{
NICOLAE BACALBASA $^{1 *}$, IRINA BALESCU ${ }^{2}$, VLADISLAV BRASOVEANU $^{3 *}$ and ALEXANDRU FLORIN ANCA ${ }^{1,4}$ \\ 1 "Carol Davila" University of Medicine and Pharmacy, Bucharest, Romania; \\ 2 "Ponderas Academic Hospital”, Bucharest, Romania; \\ 3 "Dan Setlacec” Center of General Surgery and Liver Transplantation, \\ Fundeni Clnical Institute, Bucharest, Romania; \\ ${ }^{4}$ University Emergency Hospital, Bucharest, Romania
}

\begin{abstract}
Gestational choriocarcinomas are very rare malignancies, with only few cases being reported so far. Moreover, the presence of pelvic recurrences after surgicallytreated gestational choriocarcinomas is even scarcer situations. We present the case of a 19-year-old patient who was initially submitted to surgery with preoperative diagnosis of ectopic pregnancy. At that moment a left salpingectomy was performed, and the histopathological studies revealed the presence of a left tubal gestational choriocarcinoma. The patient was submitted to adjuvant chemotherapy with methotrexate and dactinomycin. However, six months later she was diagnosed with a pelvic recurrence so she was resubmitted to surgery, debulking to no residual disease being successfully performed. The histopathological studies confirmed the presence of a recurrent tumor with gestational choriocarcinoma structure.
\end{abstract}

Association between positive $\beta$-hCG, pelvic pain and parauterine mass in a reproductive age female is most often associated with ectopic pregnancy and necessitates emergency treatment in order to stop the evolution of the pregnancy. However, $\beta$-hCG can be secreted by neoplastic cells such as malignant ovarian germ cells tumors, gestational trophoblastic disease or teratomas (1).

This article is freely accessible online.

*These Authors contributed equally to this study.

Correspondence to: Irina Balescu, "Ponderas Academic Hospital", Bucharest, Romania. Tel: +40 724077709, e-mail: irina.balescu@ ponderas-ah.ro

Key Words: Ectopic pregnancy, gestational choriocarcinoma, debulking surgery.

\section{Case Report}

A 19-year-old grade 1 patient presented for pelvic pain and vaginal bleeding; she was submitted to a transvaginal ultrasonography which demonstrated the presence of a $5 / 4 / 5 \mathrm{~cm}$ left parauterine mass in association with increased levels of $\beta$ hCG, and with an empty uterine cavity so she was counseled for either conservative or surgical treatment. She opted for surgery, so a laparoscopic exploration of the peritoneal cavity was performed. Intraoperatively the fallopian tube presented a tumoral aspect, so a left salpingectomy was performed. The histopathological studies revealed the presence of a gestational choriocarcinoma. Postoperatively the patient was submitted to adjuvant treatment with methotrexate; however, after the second administration of this agent the high serum levels of $\beta$-hCG persisted, so it was considered to be methotrexate resistant. A second line therapy with dactinomycin was initiated, two courses being administrated. At six month follow-up, higher values of $\beta$ hCG were observed so a pelvic MRI was performed. At this time the patient was diagnosed with a large pelvic tumor invading the rectosigmoidian wall so she was re-submitted to surgery, cytoreduction to no residual disease being obtained; at this moment a rectosigmoidian resection en bloc with total hysterectomy and right adnexectomy were performed in order to achieve a good local control of the disease (Figures 1-4). The anatomo-pathological studies confirmed the same histologic origin of the tumor. The postoperative course was uneventful, the patient being discharged in the sixth postoperative day. At six months follow-up she is free of recurrent disease.

\section{Discussion}

The association of a para-uterine mass in the presence of elevated $\beta$-hCG levels and empty uterine cavity in fertile women is usually strongly suggestive for ectopic pregnancy. In 
these cases, the treatment of choice varies between conservative and surgical treatment; in our case choosing the surgical approach offered the patient the chance to have an earlier diagnosis of this malignancy and a more appropriate approach (2-6). However, although a potentially curative surgical procedure was performed, the long term follow-up described the apparition of a large pelvic recurrence demonstrating the aggressive biology of this histopathological subtype.

When it comes to the gestational choriocarcinoma, this is a variant of trophoblastic tumor originating from abnormal trophoblastic hyperplasia and anaplasia with absent villi (79). It is an aggressive malignant disease characterized by a vascular pattern of spread leading to the apparition of distant metastases such as lung, brain or liver lesions (9). It can develop in association with term or preterm, with hydatiform moles or in rare situations in association with ectopic pregnancies (10). Only $0.76-4 \%$ of all reported choriocarcinomas arise from an ectopic pregnancy (11-12). Moreover, it is estimated that the incidence of gestational choriocarcinoma associated with ectopic pregnancy is of 1.5 per 1,000,000 births (13).

When it comes to the histopathological classification, gestational choriocarcinomas represent a subtype of gestational trophoblastic disease along with hydadiform mole, invasive mole and placental site trophoblastic tumors characterized by the presence of large areas of hemorrhage and necrosis. Gestational choriocarcinomas usually arise from the epithelium of the chorionic villi and is most commonly encountered inside the uterine cavity. Both gestational choriocarcinomas and epithelioid trophoblastic tumors rarely arise from ectopic pregnancies, the presenting signs and symptoms including adnexal masses, amenorrhea or irregular vaginal bleeding, abdominal pain positive pregnancy test and pelvic pain (5).

Due to the fact that most often these patients have a preoperative diagnosis of ectopic pregnancy, the first intention treatment is a fertility sparing one, limited surgical procedures being performed. Similarly, in our case, a salpingectomy was performed, the final diagnosis being established histopathologically. Once the malignant elements were revealed, the patient was submitted to adjuvant chemotherapy with synchronous $\beta$-hCG levels measurements. However, the long-term evolution was complicated by the apparition of a large pelvic recurrence imposing reoperation. At the time of the second surgical procedure, a radical resection was performed and, this time the postoperative evolution was improved, no signs of recurrent disease being revealed at six months follow-up.

Although initially gestational trophoblastic disease was considered to be associated with poor outcomes as high rates of mortality being reported, nowadays the overall prognosis has significantly improved even in widespread metastatic disease if cytoreductive surgery to no residual disease is achieved (14-16).

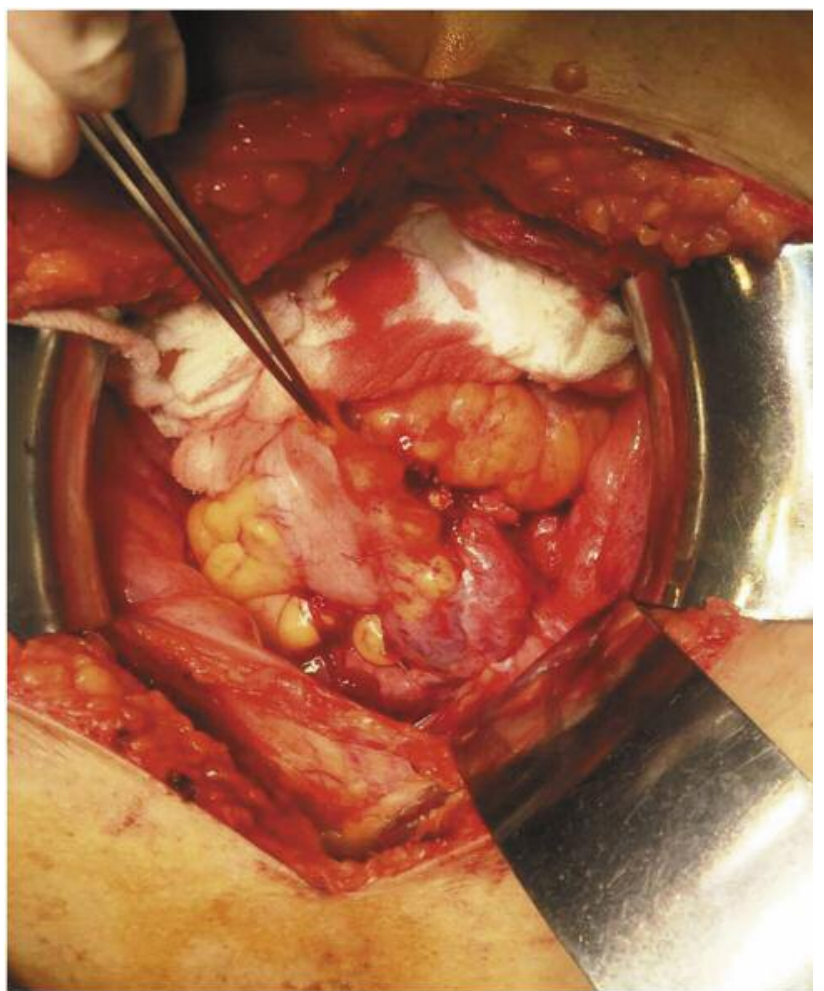

Figure 1. Initial intraoperative aspect: large pelvic tumor invading the rectosigmoidian loop.

In the study conducted by Cortes-Charry et al. and published in 2006 the authors reported a prevalence of gestational trophoblastic disease in ectopic pregnancy of 0.16 at 1,000 deliveries (six such cases being reported between 1996 and 2004). The mean age of patients was 29 years, all cases being initially submitted to surgery with a preoperative diagnosis of ectopic pregnancy, the mean gestational age at admission being eight weeks. The histopathological studies revealed the presence of a hydadiform mole in five cases, while choriocarcinoma was identified in a single case (17).

In the study conducted by Muto and published in 1991 the authors included 2,100 women with gestational trophoblastic disease, tubal location being revealed in $0.8 \%$ of cases (16 patients); among these cases, the choriocarcinoma subtype was revealed in six cases, in all cases the preoperative diagnosis being of tubal pregnancy. When it comes to the long-term evolution, four out of the six cases diagnosed with choriocarcinomas developed distant metastases (while among cases with tubal moles only one of the 10 cases was diagnosed with distant metastases) (11).

One of the largest studies conducted on the theme of gestational choriocarcinomas diagnosed during surgery for presumed cornual pregnancies came from Liu et al. and was recently published in the Journal of Cancer Biology and 


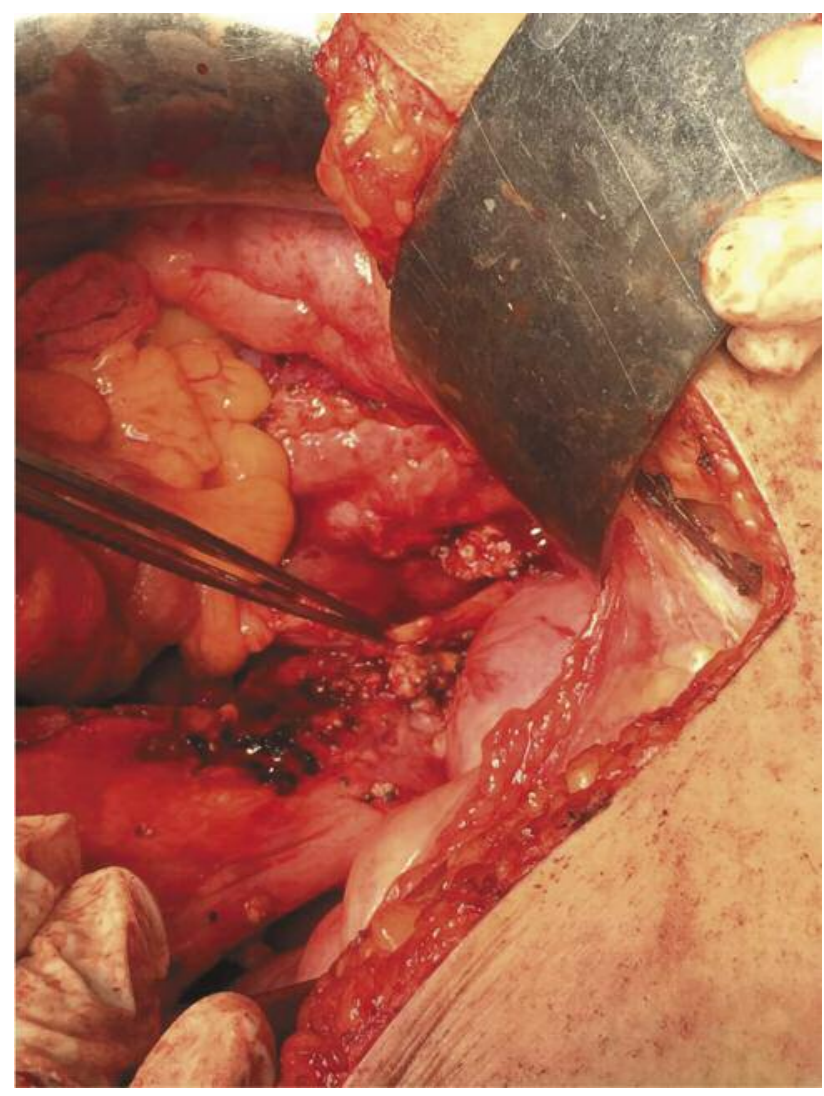

Figure 2. The aspect after dissection of the sigmoidian loop. The tumoral process invades both the uterus and the rectum.

Medicine. The study included five patients submitted to surgery with a preoperative diagnosis of tubal pregnancy between 2010 and 2014 in Peking Union Medical College Hospital. The mean age of patients was 34.6 years while the mean $\beta$-hCG level was $47,930.6 \pm 25,128.3 \mathrm{mIU} / \mathrm{ml}$. In all cases enlarged cornual areas were diagnosed intraoperatively while the histopathological studies confirmed the presence of gestational choriocarcinomas. Postoperatively all patients were submitted to adjuvant chemotherapy, a single case being resubmitted to surgery for a persistent uterine mass after the third cycle of chemotherapy. In this case hysterectomy was performed; after a mean follow-up period of 29.8 months \pm 19.1 months, no patient presented recurrent disease (18).

An interesting report comes from Kagel et al., who presented the case of a 26-year-old patient who was investigated for thoracic pain and dyspnea and who was diagnosed with a large tumoral mass of the anterior mediastinum in association with high $\beta$-hCG circulating levels. She was submitted to surgery, the tumoral mass being

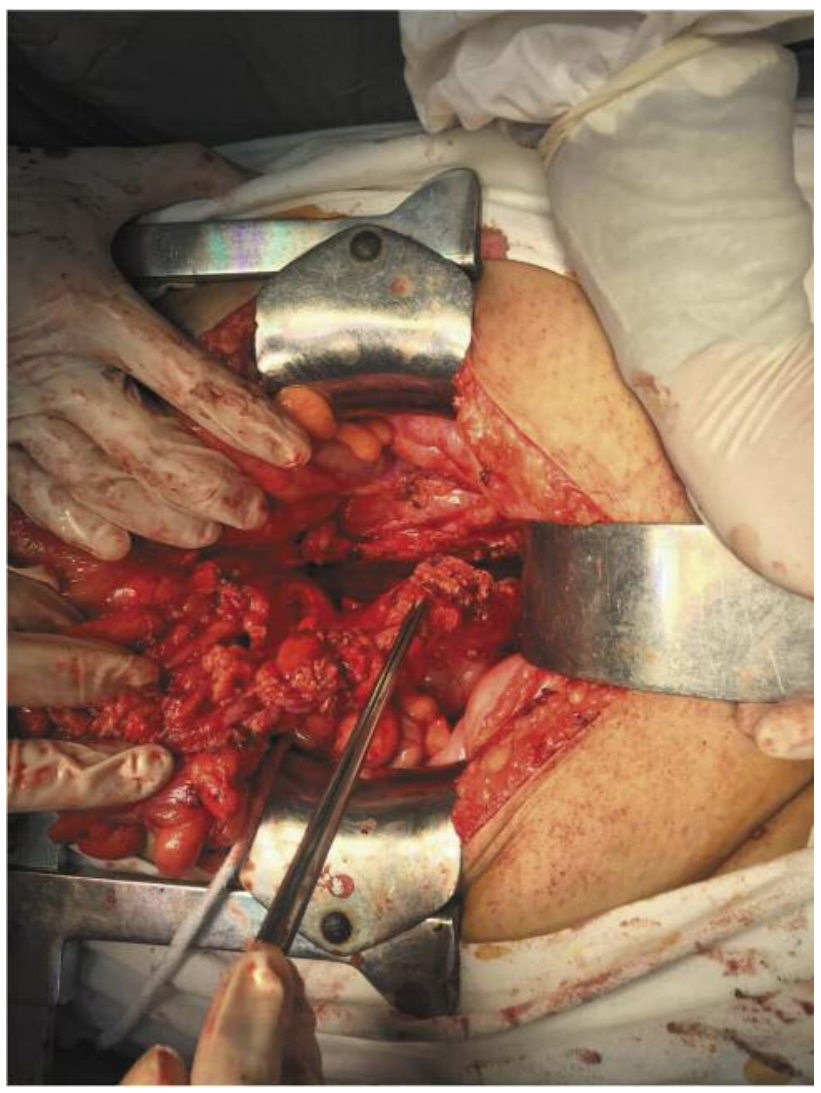

Figure 3. The aspect after tumoral mobilization en bloc with total hysterectomy and rectosigmoidian resection.

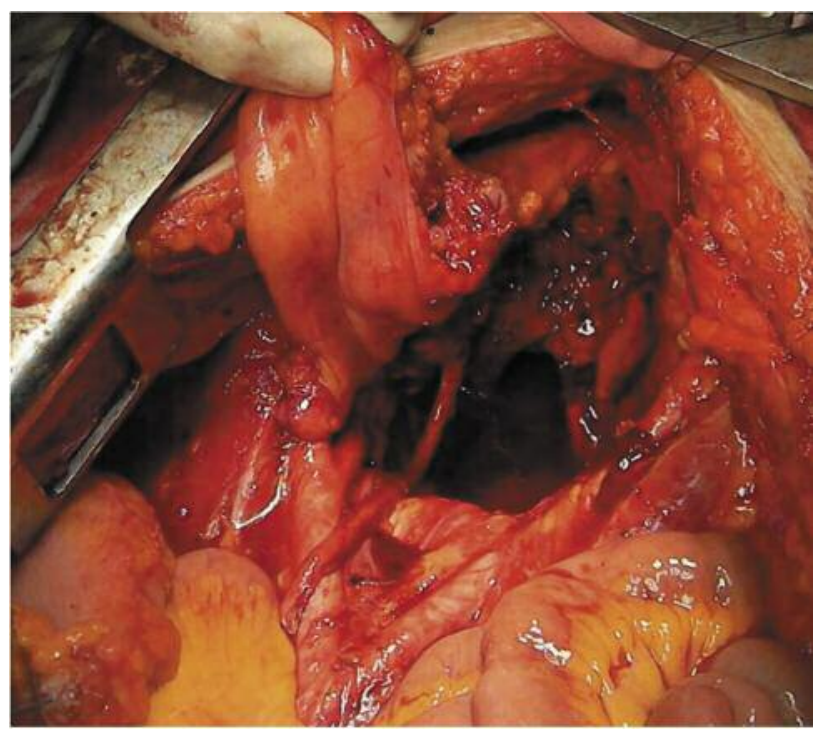

Figure 4. The final aspect after resection of the pelvic tumor en bloc with total hysterectomy and rectosigmoidian resection. 
completely resected. The histopathological studies revealed the presence of a mediastinal choriocarcinoma while the postoperative levels of $\beta$-hCG returned to normal. When analysing the medical history of the patient, a history of tubal pregnancy was found; at that moment a unilateral salpingectomy was performed, the histopathological studies infirming the presence of malignant cells. However, when comparing the microscopic aspects of the two specimens, multiple similarities were found, enabling the authors to consider that the mediastinal mass was in fact distant metastases from a tubal gestational choriocarcinoma (19).

\section{Conclusion}

Gestational choriocarcinomas associated with ectopic pregnancy are rare eventualities. The particularity of the case that we presented was the tubal location of the pathological process which was initially confounded with an ectopic pregnancy. Once the final histopathological diagnosis was established, although the patient was submitted to adjuvant chemotherapy, she developed a large pelvic recurrence, demonstrating the poor response to this approach. However, once the diagnosis or recurrent disease was revealed, she was resubmitted to surgery with curative intent, a good control of the disease being achieved.

\section{References}

1 American College of Obstetricians and Gynecologists: ACOG Practice Bulletin. Management of adnexal masses. Obstet Gynecol 110: 201-214, 2007.

2 Rivera C, Poingt $M$, Vandenbossche $F$ and Jougon J: A mediastinal germ cell tumor mimicking an ectopic pregnancy. J Gynecol Oncol 22: 288-291, 2011.

3 Dawley B, Acuna A and Grasu B: Ectopic production of HCG by a benign ovarian mature cystic teratoma simulating an extrauterine pregnancy: a case report. W V Med J 108: 15-17, 2012.

4 Ozkaya O, Sezik M, Aydin AR, Kapucuoglu N and Kaya H: Mixed germ cell tumor of the ovary mimicking unruptured ectopic pregnancy presenting with unusually high serum alphafetoprotein level. Gynecol Surg 2: 307-310, 2005.

5 Balat O, Kutlar I, Ozkur A, Bakir K, Aksoy F and Ugur MG: Primary pure ovarian choriocarcinoma mimicking ectopic pregnancy: a report of fulminant progression. Tumori 90: 136-138, 2004.

6 Kucera C, Cox-Bauer C and Miller C: Apparent ectopic pregnancy with unexpected finding of a germ cell tumor: A case report. Gynecol Oncol Rep 21: 31-33, 2017.
7 Shih IM and Kurman RJ: Epithelioid trophoblastic tumor: a neoplasm distinct from choriocarcinoma and placental site trophoblastic tumor simulating carcinoma. Am J Surg Pathol 22: 1393-1403, 1998.

8 Allison KH, Love JE and Garcia RL: Epithelioid trophoblastic tumor: review of a rare neoplasm of the chorionic-type intermediate trophoblast. Arch Pathol Lab Med 130: 1875-1877, 2006.

9 Lurain JR: Gestational trophoblastic disease I: epidemiology, pathology, clinical presentation and diagnosis of gestational trophoblastic disease, and management of hydatidiform mole. Am J Obstet Gynecol 203: 531-539, 2010.

10 Lurain JR: Gestational trophoblastic tumors. Semin Surg Oncol 6: 347-353, 1990.

11 Muto MG, Lage JM, Berkowitz RS, Goldstein DP and Bernstein MR: Gestational trophoblastic disease of the fallopian tube. J Reprod Med 36: 57-60, 1991.

12 Nayama M, Lucot JP, Boukerrou M, Collinet P, Cosson M and Vinatier D: (Tubal choriocarcinoma: a case report and review of the literature). J Gynecol Obstet Biol Reprod (Paris) 36: 83-86, 2007.

13 Gillespie AM, Lidbury EA, Tidy JA and Hancock BW: The clinical presentation, treatment, and outcome of patients diagnosed with possible ectopic molar gestation. Int J Gynecol Cancer 14: 366-369, 2004.

14 Hancock BW, Seckl MJ, Berkowitz RS and Cole LA (eds.): Gestational trophoblastic disease, 3rd ed. International Society for the Study of Trophoblastic Diseases, London, UK, 2009.

15 Soper JT: Gestational trophoblastic disease. Obstet Gynecol 108: 176-187, 2006.

16 Berkowitz RS and Goldstein DP: Current management of gestational trophoblastic diseases. Gynecol Oncol 112: 654-662, 2009.

17 Cortes-Charry R, Figueira LM, Garcia-Barriola V, Gomez C, Garcia I and Santiago C: Gestational trophoblastic disease in ectopic pregnancy: A case series. J Reprod Med 51: 760-763, 2006.

18 Liu S, Fan C, Feng F, Xiang Y, Wan X and Ren T: Prognosis of gestational choriocarcinoma diagnosed incidentally during laparoscopy for a presumed cornual pregnancy: a report of five cases. Cancer Biol Med 14: 191-193, 2017.

19 Kagel T, Lemburg SP, Muller KM, Laczkovics A, Nicolas V and Heyer CM: (Mediastinal metastasis of a tubal choriocarcinoma following ectopic pregnancy as a rare cause of thoracic pain). Zentralbl Gynakol 128: 90-94, 2006.
Received October 5, 2017

Revised November 5, 2017

Accepted November 5, 2017 